\title{
MEDIOS DE COMUNICACIÓN Y CULTURA DE PAZ PARA LA CONVIVENCIA DEMOCRÁTICA
}

\author{
MEDIA AND CULTURE OF PEACE FOR DEMOCRATIC COEXISTENCE
}

\author{
Ana Julia Hernández-Milla ${ }^{1} *$ (D). \\ 1. Escuela Judicial del Estado de Tabasco, México. anajulia_73@hotmail.com
}

* Autor de correspondencia: Ana Julia Hernández-Milla, correo electrónico: anajulia_73@hotmail.com

\section{RESUMEN}

El desarrollo de las sociedades dentro de un orden establecido considera una cultura basada en las normas y obligaciones competentes al ciudadano, estructuras sociales e instituciones del sistema de justicia. En ese sentido, potencializar los medios de comunicación para asegurar una sociedad con cultura de paz reflejada en una convivencia democrática, involucra a diversos actores con un fin de bien común, que de forma directa e indirecta beneficie a otros sectores o ámbitos de la población e instituciones. Este documento pretende dar un panorama de lo benéfico que resulta socializar, interactuar e innovar mecanismos de acción que preponderen a una sociedad sostenible, democrática e integral, basada en la participación, pluralidad, tolerancia y respeto, teniendo como finalidad realizar reflexiones sobre la importancia de la implementación de la cultura de paz como mecanismo que permita favorecer y promover valores cívicos y sociales, en la construcción de una eficaz convivencia democrática y por ende la prevención de la violencia, incluida la política, y el rol importante que tienen los medios de comunicación, como ente de procesamiento de información oportuna a la ciudadanía, que muchas veces sobrepasan la intención de informar, para influir en las simpatías o ánimos, "contrario sensu", confrontando a actores políticos y sus simpatizantes.

Palabras clave: convivencia inclusiva; Estado; educación; paz; sociedad civil.

Cómo citar:

Hernández-Milla, Ana Julia. (2021). Medios de comunicación y cultura de paz para la convivencia democrática. Revista de Investigaciones Universidad del Quindio, 33(S2), 24-28. https://doi. org/10.33975/riuq.vol33nS2.607 


\begin{abstract}
The development of societies within an established order considers a culture based on the norms and competent obligations to the citizen, social structures and institutions of the justice system. In this sense, empowering the communication media to ensure a society with a culture of peace reflected in a democratic coexistence, involves various actors with a goal of common good, which directly and indirectly benefits other sectors or areas of the population and institutions. This document aims to give an overview of how beneficial it is to socialize, interact and innovate action mechanisms that prevail in a sustainable, democratic and integral society, based on participation, plurality, tolerance and respect, with the aim of reflecting on the importance of the implementation of the culture of peace as a mechanism that allows to favor and promote civic and social values, in the construction of an effective democratic coexistence and therefore the prevention of violence, including politics, and the important role of the media, as a body for the processing of timely information to citizens, which often go beyond the intention of informing, to influence sympathies or spirits, "contrary sensu", confronting political actors and their supporters.
\end{abstract}

Keywords: inclusive coexistence; State; education; peace; civil society.

\title{
INTRODUCCIÓN
}

En una sociedad en constante cambios sociales, políticos, culturales, económicos, ambientales, se configuran nuevas dinámicas en las sociedades. Ante esos cambios, surgen o manifiestan conflictos que pueden desencadenar actos de violencia. Usualmente el conflicto está visto de forma negativa ya que las partes involucradas se radicalizan sin poder considerar el diálogo y comunicación para resolver el problema.

En un contrato social en que estamos por lo menos en el estado mexicano, en el que dicho contrato las partes son el Estado y la ciudadanía; se debe considerar involucrar acciones de acción participación aplicando mecanismos que impacten de forma local a lo global.

Por ello, este documento pretende describir la importancia de considerar a través de los medios de comunicación una cultura de paz para una convivencia democrática, capaz de plantear acciones a un corto y mediano plazo para tener resultados benéficos, sostenible e integral como sociedad.

Es de señalar, que no sólo tiene impacto de forma individual sino colectiva, es ahí que desde el escenario micro las personas puedan contribuir desde su ámbito laboral, familiar, comunitario; los espacios públicos son infraestructura que permite la concentración y socialización de valores entre los individuos, por lo tanto, el mantenimiento de los mismos implica una inversión en procesos de bienestar ciudadano.

\section{METODOLOGÍA}

En el reflejo de procesos transformativos en las sociedades resulta complejo de comprender e identificar aquellos factores que propician situaciones de conflicto en el escenario político. En ese sentido, para rescatar o asegurar la convivencia entre ciudadanos y sus instituciones, hay que considerar los derechos 
y obligaciones que como sociedad tenemos. En ese sentido, los medios de comunicación representan una plataforma de divulgar acciones específicas en las que participe la sociedad civil, se integre el sector público y privado.

La realidad concreta, resulta ser cada vez más alarmante y en ese proceso de rescate o estabilidad que se desea de la convivencia (Carozzo, 2017), nos debemos percatar de tener acceso y garantía a aquellas instancias que dentro de sus funciones y competencias está atender, mitigar, prevenir conflictos.

Ante está situación este documento versa en un enfoquecualitativoconalcance descriptivo einterpretativo con técnica documental. Se realizó una búsqueda y consulta de fuentes bibliohemerográficas que nos permitió distinguir elementos categóricos que se exponen en líneas siguientes.

\section{RESULTADOS}

\section{Medios de comunicación y cultura de paz}

Para establecer muy claramente la situación de la importancia de los medios de comunicación en la construcción de una convivencia democrática, el Instituto Nacional Electoral, considera que por una falta de cultura democrática (CLADE, OREALC, UNESCO, 2013) y a la vez de herramientas de educar para la paz, los índices de violencia política han ido en aumento.

En México, las redes sociales, y los medios de comunicación tradicional, generan información en donde no se fomentan valores de paz positiva, ni valores para formar nuevos ciudadanos con educación cívica (Hernández et al., 2017), dichas publicaciones ejercen violencia política sin ninguna distinción, se puede constatar en el informe anual que hace el INE sobre el estudio de la información que se maneja en los medios de comunicación durante los procesos electorales. De dicho informe se encontró que en el Proceso Electoral Local 2018-2019, en redes sociales se presentaron 25 casos que se clasificaron como positivos porque precisamente permiten visualizar la violencia, mientras que en prensa tradicional se presentaron 12 .

Es por ello, que es necesario, tomar en consideración la implementación de acciones que fomenten la convivencia democrática, con apoyo de los medios de comunicación que permitan al ciudadano tomar conciencia en el respeto de sus derechos y obligaciones dentro de la sociedad, vivir con el que piensa distinto, ya sea por su idioma, cultura, raza, religión, ideología política, etc., en armonía, buscando formas dignas, honestas e igualitarias para que las personas puedan expresarse libremente y participar en la vida democrática del país, respetando el derecho de los demás. La Cultura de Paz, por otro lado, se basa en principios que respetan los derechos humanos, la democracia y la tolerancia, la educación para la paz, la libre circulación de información, creando condiciones propicias para establecer la paz y consolidarla.

Naciones Unidas (1998) la describe como aquella que "consiste en una serie de valores, actitudes y comportamientos que rechazan la violencia y previenen los conflictos tratando de atacar sus causas para solucionar los problemas mediante el diálogo y la negociación entre las personas, los grupos y las naciones" considera un Estado alterado desde un escenario micro y macro en diversos ámbitos, por destacar el político ya que permea en estructuras de poder y toma de decisiones. Al respecto, es necesario analizar, la violencia política, ¿qué es, como se produce y manifiesta en los medios de comunicación?, para que coadyuve la cultura de paz, en los procesos necesarios, poniendo en práctica 
formas de interacción a nivel personal y colectivo entre las personas para la construcción de una digna convivencia democrática.

Esta forma de violencia, consiste sencillamente en que un actor o actora pretende dañar o generar efectivamente un daño a una persona física o colectiva durante el ejercicio de sus derechos políticos, que pueden incluir desde calumnias hasta el asesinato de una candidata o candidato. A pesar de la notable violencia en el informe del proceso electoral local 2018-2019 del INE "de cada cien publicaciones se destacó que 21 incluyeron un tipo de violencia política, demostrando la falta de cultura educativa para la paz y la importancia de generar mecanismos eficaces para una convivencia democrática" (INE, 2021), además de ello, considerar la obligación que como sociedad civil se debe fortalecer.

Uno de los factores importantes de la intervención para la formación en convivencia democrática y cultura de paz en las estructuras debe comprender el plano programático (UNESCO, 2008), su función como estructura y formas de implementación, así como los roles e interacciones que se construyen en la propia esfera de la sociedad.

\section{Convivencia democrática}

La convivencia democrática tiene que ver con la "experiencia de participar en la vida compartida. Refiere al desarrollo de capacidades reflexivas y de herramientas para trabajar con otros, para resolver los conflictos de manera pacífica y establecer los acuerdos que regulen la vida en común" (Fierro, et al., 2010). Parte del principio de comunidad y bien común a partir del diálogo, teniendo como características:

- Manifestar de manera asertiva opinión, acción y pensamiento.

- El respeto mutuo. Respecto a las críticas, estás deben pronunciarse y aceptarse de forma constructiva.

- Desde la perspectiva del bien común realizar las tomas de decisiones.

- Gestionar y solucionar el conflicto desde el diálogo y comunicación.

Estas características generales para una convivencia democrática, dispone de cooperación entre la sociedad civil y el Estado. En el caso de conflictos o diferencias políticas, es importante considerar el papel que juegan los medios de comunicación con la divulgación de ataques entre partidos, militantes y la sociedad civil. En lugar de poder ser los medios de comunicación una vía certera de difusión de propuestas se ha alineado a divulgar ofensas que repercuten en la dinámica social y política de la sociedad al mismo tiempo de alterar el orden.

La educación para la convivencia democrática puede situarse como aquella "educación que se ofrece para enseñar y promover el desarrollo de conocimientos, valores y destrezas necesarios para vivir en una sociedad democrática (Educación para la democracia o para la vida en democracia)" (Rodino, 2003). Lo que nos lleva a considerar la educación como un mecanismo eficaz para afianzar la convivencia de forma equitativa e igualitaria.

El aspecto de la democracia en el sistema político representa un criterio en construcción, en la que participan los partidos políticos, sociedad civil, gobernantes, representantes, visto desde el escenario de género o grupo vulnerables, implica considerar un lenguaje inclusivo no discriminatorio.

Además, establecer una toma de decisiones que considere de manera fundamental no perjudicar o 
afectar al colectivo (Pérez, 2008), por lo que debe recaer en equitativa, participada y ejecutada.

La convivencia democrática distingue una forma de actuar propia de las estructuras de la sociedad y la propia sociedad civil, edifica un Estado democrático con apertura y tolerancia en las acciones estratégicas. Coadyuva a un sistema de acceso a la información, transparencia y rendición de cuenta (Berdeja et al., 2020) por parte de las instituciones, dando la pauta para mejorar las condiciones de vida.

\section{DISCUSIÓN}

"La convivencia democrática precisa fomentar la generalización de una educación para todos y a lo largo de toda la vida que se inspire en valores y se alimente en una visión global de un mundo en paz y libertad" (Díez, 2002).

A partir de la educación formal e informal se conforman procesos transicionales como sociedad y en el propio quehacer del sistema y administración de justicia que comulguen con la creación y estabilidad de la convivencia democrática a partir de la solidaridad, respeto a los derechos humanos, promoción de cultura de paz, lenguaje inclusivo y resolución de conflictos.

La convivencia democrática nos determina como una sociedad organizada capaz de identificar, procesar aspectos que lleve de forma progresiva a una sociedad de esa índole. Los medios de comunicación son un mecanismo estratégico e innovación para interactuar desde diversos aspectos, los cuales en el estado mexicano requieren de mayor impulso.

\section{REFERENCIAS}

1. Berdeja Rivas, M., Martínez Prats, G., \& Silva Hernández, F. (2020). Transparencia y acceso a la información pública ante situaciones de pandemia. Publicaciones E Investigación, 14(2). https:// doi.org/10.22490/25394088.4351

2. Carozzo Campos, Julio César. (2017). Dimensiones de la convivencia. Revista del Observatorio sobre la Violencia y Convivencia en la Escuela, 3(5), 27-42.

3. CLADE, OREALC, UNESCO. (2013). Informe regional. Consulta sobre la educación para la convivencia democrática y derechos humanos. Campaña Latinoamericana por el Derecho a la Educación, Brasil.

4. Díez Hochleitner, Ricardo. (2021). Educar para la convivencia democrática. El País, México.

5. Fierro C., Carbajal, P. y Martínez-Parente, R. (2010). Ojos que sí ven. Casos para reflexionar sobre la convivencia en la escuela ( $2^{\mathrm{a}}$ reimpresión). México: SM.

6. Hernández Arteaga, José Alberto, Luna Hernández, José Alberto \& Cadena Chala, Martha Cecilia. (2017). Cultura de paz: una construcción educativa aporte teórico. Revista Historia de la Educación Latinoamericana, 19(28).

7. INE. (2021). Violencia política de género en la comunicación política durante el proceso electoral local 2018-2019. México.

8. Naciones Unidas. (1998). Resolución A/52/13. Cultura de Paz.

9. Pérez Jiménez, César. (2008). La convivencia social como proyecto político colectivo. Utopía y Praxis Latinoamericana, 13(42), 107-129.

10. Rodino, A. (2003). Educación para la vida en democracia: Contenidos y orientaciones metodológicas. Instituto Interamericano de Derechos Humano: San José.

11. UNESCO. (2008). Convivencia democrática, inclusión y cultura de paz. Lecciones desde la práctica educativa innovadora en América Latina. Pehuén. Chile. 